\title{
Erratum
}

\section{Non-Empirical Interpretation of Nitrogen 14 Nuclear Quadrupole Coupling Constants}

\author{
E. Kochanski, J. M. Lehn, B. Levy \\ Institut de Chimie, Université Louis Pasteur, 1, rue Blaise Pascal, Boîte postale 296/R8, F-67008 \\ Strasbourg Cedex, France
}

Theoret. Chim. Acta (Berl.) 22, 111-129 (1971)

Some interchanges have occurred in the ordering of the $\chi_{i i}$ axes on conversion of the reported experimental values of the $\chi_{i i}$ components to the axis systems used in the above paper. The correct ordering of the experimental $\chi_{i i}$ values requires to exchange the $\chi_{x x}$ and $\chi_{z z} \exp (\mathrm{MW})$ values for compounds V and VI and to exchange the $\chi_{Y Y}$ and $\chi_{z Z} \exp (\mathrm{MW})$ values for compound $I$.

Also: the axes given on page 115 refer to compounds $\mathrm{I}-\mathrm{V}$; read in Table 1, compound IX trans: (at $\mathrm{N}(1)): \theta=-59^{\circ} 16^{\prime}(y)$.

Received September 5, 1979 